\title{
Una mirada al rediseño curricular de los planes de estudios de la Escuela de Ciencias de la Educación de la Universidad Estatal a Distancia
}

\author{
Xinia María Calvo Cruz \\ Asesora Curricular.UNED
}

Recibido: 6 de abril de 2010

\section{RESUMEN}

Este artículo presenta los resultados de la asesoría ofrecida por el Programa de Apoyo Curricular y Evaluación de los Aprendizajes (PACE), en el año 2009, a las Comisiones de Autoevaluación (CAES) de la Escuela de Ciencias de la Educación de la Universidad Estatal a Distancia, con el fin de lograr el rediseño de los planes de estudio de los diferentes programas. Para lograr el objetivo planteado se mantuvo constante coordinación con las CAES, ofreciendo la guía curricular requerida. Para esta experiencia la asesora curricular elaboró guías de apoyo para el trabajo de cada uno de los apartados del plan de estudios. También se estableció un periodo de entrega de avances y revisiones y se mantuvo constante apoyo de la Directora de la Escuela. Cada uno de los programas concretó su nueva propuesta de plan de estudios, como parte del plan de mejoras del informe con fines de acreditación o mejoramiento continuo.

\section{PALABRAS CLAVE}

Calidad, centros y programas de apoyo, acreditación, mejoramiento continuo, pertinencia.
Aceptado: 30 de setiembre de 2010

\section{ANTECEDENTES}

La Universidad Estatal a Distancia (UNED) se crea en 1977, bajo la administración del Licenciado Daniel Oduber Quirós (1974-1978) y bajo la tutela de Fernando Volio Jiménez, como Ministro de Educación. Es el Licenciado Volio quien promueve la idea de creación de una institución de educación superior en Costa Rica, una vez que regresó de España de una reunión de Ministros de Educación, 
en la cual el Sr. Juan Diez Nicolás, exrector de la Universidad Nacional de Educación a Distancia de España, narró la experiencia de la institución que representaba (CIDRED, 2005).

Según la Ley de Creación y las Políticas Universitarias, lo que hoy conocemos como Escuela de Ciencias de la Educación, inicialmente era una sección del área de Humanidades. Sin embargo sólo un año después,

"....al integrarse las diferentes áreas para facilitar los procesos de coordinación, la Escuela se constituyó como área de Educación. Una década después y al calor de la Reforma Universitaria de 1989, se configura la actual Escuela de Ciencias de la Educación, con una estructuración por cátedras y programas, que le dieron una mayor independencia y libertad de acción para impulsar nuevas carreras y otros programas." (UNED, 2010,sp)

Hoy en día, la Escuela de Ciencias de la Educación cuenta con nueve programas de grado y además ofrece cuatro programas de maestría y un Doctorado en Educación.

\section{- La calidad, un compromiso de la UNED}

Ciertamente, la Universidad ha tenido desde siempre el estandarte de la calidad y el compromiso con la sociedad, así se lee desde los objetivos y funciones de la UNED, establecidos en 1977 y publicados por primera vez en La Gaceta No.50 del 12 de marzo de 1977.

En 1979, sólo dos años después de sus inicios, la UNED contaba con el Centro de Control Académica (CONCAL), el cual se destacó por la valoración de unidades didácticas, tutorías y evaluaciones académicas en general (Azze, 2007).

Honrando su compromiso de ofrecer calidad, en 1993 la Universidad firma, como parte del Consejo Nacional de Rectores de Universidades
Públicas (CONARE), el convenio de creación del Sistema Nacional de Acreditación de Costa Rica (SINAES), comprometiéndose con la excelencia, la evaluación y el perfeccionamiento permanente (Azze, 2007).

En el año 1997, se crea el Centro de Investigación y Evaluación Institucional (CIEI), instancia que tuvo a cargo el iniciar la formulación de los primeros documentos en materia de autoevaluación de programas en la UNED. Valga subrayar que es desde este centro donde surge la primera Guía de Autoevaluación de Carreras (Azze, 2007).

Más adelante se continúa unificando esfuerzos desde directrices, congresos, entre otros medios que posibilitaran el ofrecer programas pertinentes a las necesidades de la sociedad. De estos esfuerzos surge la decisión de crear en el año 2002 el Centro de Mejoramiento de los Procesos Académicos (CEMPA) y el programa de Autoevaluación Académica (PAA), a la vez que se liberó al CIEI de la evaluación académica y se le atribuyó exclusivamente la evaluación institucional.

Desde entonces, el PAA se concibe como la unidad encargada de la autoevaluación de carreras y programas para fines de mejoramiento de la calidad y de la acreditación.

En el 2006, fruto de algunas mociones que surgieron del Tercer Congreso Universitario-2006 "Por el fortalecimiento de una academia con calidad", el CEMPA se reestructura e inician tanto el Programa de Investigación en Fundamentos de Educación a Distancia (PROIFED) como el Programa de Apoyo Curricular y Evaluación de los Aprendizajes (PACE).

A la fecha, el PACE se ocupa de todo lo relacionado con el currículo $y$, en coordinación con el PAA, asesora y acompaña a las Comisiones de Autoevaluación del programa que por decisión propia se someten al proceso de autoevaluación en miras de la acreditación o la mejora continua. 


\section{- La Escuela de Ciencias de la Educación en proceso de acreditación y lo mejora continua}

En el año 2004, la Licenciatura en Ciencias de la Educación con énfasis en I y II Ciclo, se aventura en el proceso de autoevaluación, se constituyó así en el primer programa en la Escuela que surcaba un camino desconocido para todos. De este esfuerzo surgió un Informe de Autoevaluación, que más tarde truncaría los siguientes pasos al obtener la negación de la continuidad del proceso por no ser aceptado en la agencia acreditadora, SINAES. Con este esfuerzo se perdió la ilusión y un monto elevado monto económico; lo que sin duda generó resistencia de los programas para aventurarse hacia esta meta.

Ante este panorama, la Escuela de Ciencias de la Educación inicia un proceso de letargo en la materia; no es hasta el 2008 cuando se reactiva un proceso de las mismas características y el programa de Informática Educativa tiene como resultado la acreditación de su carrera en el 2009, constituyéndose en la primera carrera de la Escuela acreditada con el sistema nacional SINAES.

Al igual que Informática Educativa inicia su proceso de autoevaluación, los otros programas unieron esfuerzos e iniciaron los procesos de autoevaluación, con miras a la acreditación o mejora continua.

De esta forma, en el 2009 el PACE abocó esfuerzos para brindar el apoyo y el acompañamiento curricular sistematizado; algunos de los programas se habían adelantado en el proceso, producto de la reflexión de los encargados de programa, de cátedra y los tutores.

Con el fin de ofrecer guía oportuna, se planificó una estrategia de trabajo que permitiera a la asesora la atención de todos y cada uno de los equipos. Valga subrayar que algunos programas no habían sido analizados y cambiados desde sus inicios, sino que fueron "maquillados curricularmente" en la práctica, pero esas buenas intenciones de los encargados no se sistematizaron en un documento escrito; lo que resultaba en que la mayoría de ellos ofertaba un plan que no correspondía a su perfil y estructura curricular.

Así la situación, la directora de la Escuela de Ciencias de la Educación asumió el compromiso, en coordinación con la asesora curricular, de unificar esfuerzos y abrir espacios dedicados exclusivamente a la reflexión desde lo interno de cada una de las Comisiones de Autoevaluación Académica. Como fruto de esta idea, se realizó una serie de encuentros destinados a la asesoría curricular en materia del diseño o rediseño de planes de estudio.

La experiencia acumuló 16 guías que contenían orientaciones para la concreción de cada uno de los apartados del plan de estudio. Como complemento de la dinámica se asumió la idea de trabajar por tareas y enviar los productos parciales a su respectiva revisión, para así ir generando el documento final.

En todo este trabajo fue de suma importancia lo que se denominó "Validación de perfil", ya que en este espacio se convocó a especialistas externos o internos y/o a veces grupos mixtos, representantes de las otras universidades públicas que ofrecen las diversas carreras, docentes en ejercicio, tutores, estudiantes, entre otros, para presentarles el objeto de estudio, los objetivos generales y específicos, los ejes transversales, las áreas disciplinarias, el perfil profesional de salida, la estructura y la malla curricular. Todo lo anterior, fue validado en una dinámica de sana discusión académica, donde se recibieron los insumos y se valoraba la pertinencia de su incorporación al documento en proceso o bien, se desestimaba después de una reflexión de la Comisión de Autoevaluación y la asesoría curricular.

Este espacio generó mucho aporte, puesto que se tuvo la oportunidad de compartir la visión de cada participante desde su ámbito de acción, con un único objetivo: el formar al docente que 
requiere la sociedad desde los diferentes objetos de estudio.

\section{- Reseña de cada plan de estudios rediseñado}

La Licenciatura en Educación General Básica I y II Ciclos, con énfasis en necesidades educativas especiales, fue rediseñada con fines de mejoramiento, puesto que ya estaba aprobada por CONARE, pero aún sin salir a oferta, se detectó que su objeto de estudio se estaba traslapando al del Bachillerato en Educación Especial. En el proceso de rediseño participaron tanto evaluadores externos como internos que validaron y avalaron la nueva propuesta.

El Diplomado, el Bachillerato y la Licenciatura en Preescolar inician su autoevaluación en el año 2007, en materia curricular se planteó un plan de mejoras y a la fecha se ultiman detalles de su informe de autoevaluación con miras a la acreditación.

En relación con el Bachillerato en Educación Especial, en el 2006 da inicio el proceso de autoevaluación, que concluye en el 2009. Actualmente, el equipo coordina con el PAA para concluir el informe de autoevaluación en aras de acreditación.

En el caso de la Licenciatura en Educación Especial, es importante anotar que se encuentra en proceso de construcción, se ha optado por el enfoque de competencias, lo que ha constituido un esfuerzo tanto del programa como a nivel institucional.

Como resultado del proceso de autoevaluación que inició en el 2006, también la Licenciatura en Educación Cívica presenta su propuesta del nuevo plan de estudios, como parte del mejoramiento continuo. Valga aclarar que como resultado del proceso se amplió el objeto de estudio y hoy día se plantea una Licenciatura en Estudios Sociales y Cívica.
La Licenciatura en Docencia inicia el proceso de autoevaluación en el año 2002. Para entonces, la acreditación no se creyó conveniente, por lo que inició un estudio profundo y minucioso del perfil docente. Actualmente, el plan de estudios se encuentra rediseñado en su totalidad, con un planteamiento de estructura curricular por módulos y con una propuesta metodológica virtual.

El programa de Bachillerato y Licenciatura en Administración Educativa ha tenido la oportunidad de experimentar dos procesos de autoevaluación; uno en el año 2006 como parte del mejoramiento continuo y otro en el 2009 con miras a la acreditación. Parte de los resultados de este último es la propuesta del nuevo plan de estudios y la redacción del informe de autoevaluación para SINAES.

La autoevaluación en el 2004 del Diplomado, el Bachillerato y la Licenciatura en Educación General Básica I y II Ciclo, alarmó sobre la necesidad de rediseñar la carrera, puesto que las nuevas demandas en materia de formación sobrepasan a luces lo establecido a esa fecha como currículo sistematizado. En este momento, el programa se encuentra en los últimos esfuerzos de revisión para ser presentado al Consejo de Escuela y continuar con las dependencias internas y externas para su aprobación.

\section{CONCLUSIONES}

La Escuela de Ciencias de la Educación logró rediseñar todos los planes de estudio ${ }^{1}$; cada plan apuesta a la innovación y pertinencia en su campo de acción, al planteamiento de su nuevo perfil y estructura curricular, así como a la propuesta de variados proyectos de extensión e investigación.

Así bien el trabajo curricular continúa ya que actualmente se desarrolla el primer taller de diseño y rediseño de cursos y se perfila uno por cuatrimestre. De esta forma, las diferentes estructuras curriculares irán cumpliendo 
sus proyecciones $y_{1}$ en un término no mayor de cinco años, todos los programas se estarán ofertando con diseños y materiales didácticos acordes con los requerimientos de su área; con el compromiso de que -transcurridos 5 años de oferta- nuevamente dará inicio el proceso de autoevaluación, lo cual establecerá esta práctica como cíclica y permitirá la acreditación o el mejoramiento continuo de los programas de la Escuela de Ciencias de la Educación.

\section{NOTAS}

1. El rediseño de los planes de estudio se cumplió gracias al trabajo de las diferentes comisiones de autoevaluación, al apoyo y acompañamiento de la asesora curricular designada en la Escuela y a la guía de la Máster Ida Fallas Monge, actual Ex Directora de la Escuela de Ciencias de la Educación.

\section{REFERENCIAS}

Azze, A. (2007). Aseguramiento de la calidad en universidades a distancia. El caso de la Universidad Estatal a Distancia (UNED) en Costa Rica. Revista CINDA. Acreditación y dirección estratégica para la calidad en las universidades. Colección Gestión Universitaria. Primera Edición. Santiago, Chile.

La Gaceta, N 50 (1977, 12 de marzo). Ley de creación de la Universidad Estatal a Distancia. Decreto de la Asamblea Legislativa No. 6044. San José, Costa Rica: Imprenta Nacional.

UNED. (2005). Centro de Información, Documentación y Recursos Bibliográficos. San José, Costa Rica: EUNED.

UNED. (2010). En: http://www.uned.ac.cr/educacio/conozca_laescuela.shtml 
